\title{
Whole-body magnetic resonance imaging in children - how and why? A systematic review
}

\author{
Pia Zadig ${ }^{1,2}$ (1) Elisabeth von Brandis ${ }^{3} \cdot$ Regina Küfner Lein ${ }^{4} \cdot$ Karen Rosendahl ${ }^{1,2}$ • \\ Derk Avenarius $^{1,2} \cdot$ Lil-Sofie Ording Müller ${ }^{5}$
}

Received: 31 December 2019 / Revised: 3 April 2020 / Accepted: 20 May 2020 / Published online: 25 June 2020

(C) The Author(s) 2020

\begin{abstract}
Whole-body magnetic resonance imaging (MRI) is increasingly being used for a number of indications. Our aim was to review and describe indications and scan protocols for diagnostic value of whole-body MRI for multifocal disease in children and adolescents, we conducted a systematic search in Medline, Embase and Cochrane for all published papers until November 2018. Relevant subject headings and free text words were used for the following concepts: 1) whole-body, 2) magnetic resonance imaging and 3) child and/or adolescent. Included were papers in English with a relevant study design that reported on the use and/ or findings from whole-body MRI examinations in children and adolescents. This review includes 54 of 1,609 papers identified from literature searches. Chronic nonbacterial osteomyelitis, lymphoma and metastasis were the most frequent indications for performing a whole-body MRI. The typical protocol included a coronal STIR (short tau inversion recovery) sequence with or without a coronal T1-weighted sequence. Numerous studies lacked sufficient data for calculating images resolution and only a few studies reported the acquired voxel volume, making it impossible for others to reproduce the protocol/images. Only a minority of the included papers assessed reliability tests and none of the studies documented whether the use of whole-body MRI affected mortality and/or morbidity. Our systematic review confirms significant variability of technique and the lack of proven validity of MRI findings. The information could potentially be used to boost attempts towards standardization of technique, reporting and guidelines development.
\end{abstract}

Keywords Adolescents $\cdot$ Children $\cdot$ Indications $\cdot$ Magnetic resonance imaging $\cdot$ Systematic review $\cdot$ Technique $\cdot$ Whole-body magnetic resonance imaging

Electronic supplementary material The online version of this article (https://doi.org/10.1007/s00247-020-04735-9) contains supplementary material, which is available to authorized users.

\section{Pia Zadig}

pia.karin.karlsen.zadig@unn.no

1 Department of Radiology, University Hospital of North Norway, Sykehusvegen 38, 9019 Tromsø, Norway

2 University of Troms $\varnothing$ - The Arctic University of Norway, Tromso, Norway

3 Department of Radiology, Oslo University Hospital, Oslo, Norway

4 Medical Library, University of Bergen, Bergen, Norway

5 Department of Radiology and Intervention, Unit for Paediatric Radiology, Oslo University Hospital, Oslo, Norway

\section{Introduction}

Whole-body MRI is a relatively new assessment tool that allows for imaging of the entire body in one scan, with the potential to provide both anatomical and functional information. Without the use of ionizing radiation, it enables the depiction of disease at an early stage, sometimes even before the onset of clinical symptoms. This ability to detect clinical silent lesions is often highlighted as an important feature of wholebody MRI [1-6]. The value of whole-body MRI as an advanced method to evaluate disease in adults has been investigated by several authors [7-10]; however, the results drawn from these studies are not directly applicable to children. For example, when searching for bone diseases, previous research has shown that MRI findings in the wrist and feet believed to represent pathology in the mature skeleton merely represent normal, growth-related changes in children [11-17]. Knowledge of normal signal changes in paediatric bone 
marrow is crucial for the interpretation of whole-body MRI to avoid false-positive lesions and thereby overstaging of disease, particularly when searching for clinically silent lesions. To our knowledge, no studies addressing normal bone marrow signal changes on paediatric whole-body MRI have been published. Moreover, studies addressing the precision, accuracy and clinical validity of whole-body MRI in children are sparse [18].

Despite these shortcomings, whole-body MRI is increasingly being used in children with suspected chronic nonbacterial osteomyelitis, Langerhans cell histiocytosis and malignancies, amongst others $[19,20]$. Even though the wholebody MRI protocol should, to some extent, be tailored to the specific indication, a standard whole-body MRI protocol can be used for most practical purposes and indications. At least minimum requirements for parameters (e.g., slice thickness) can be defined. Protocols seem to vary significantly across institutions, with respect to sequences, planes and imaging parameters and there is no uniform or standard protocol for whole-body MRI imaging [19-21]. Further, the nomenclature regarding anatomical coverage in these examinations is inconsistent $[20,22,23]$ and a unifying interpretation and reporting system is lacking.

Several reviews on paediatric whole-body MRI address potential indications and sequences used [19-21, 24-26], however, hitherto, no systematic review has been published. We therefore aimed to provide a systematic review of the literature to describe common indications and protocols used to assess multifocal disease, and to examine the diagnostic value of whole-body MRI in children and adolescents.

\section{Materials and methods}

\section{Information sources and search}

We conducted literature searches in Medline, Embase and Cochrane for published papers about whole-body MRI of children and adolescents until November 2, 2018. Relevant subject headings and free text words were used for the following concepts: 1) whole-body, 2) magnetic resonance imaging and 3) child and/or adolescent. The detailed search strategies are given in Online Supplementary Material 1.

\section{Study selection}

Title and abstracts of all identified papers were read by two radiologists (P.Z., a radiology resident, and E.v.B., with 10 years of experience) and checked for eligibility. The selected papers from this phase plus two papers identified through other sources were read in full text by the same radiologists and scored as being eligible for inclusion, not eligible or uncertain. In cases of disagreement or uncertainty, consensus was obtained by discussion.

\section{Criteria for inclusion and exclusion}

Studies reporting on the use and/or findings from whole-body MRI examinations to assess multifocal disease in children and adolescents up to 19 years old were included. For studies including both children/adolescents and adults, they were included if data on at least 10 children/adolescents could be identifiable separately. Studies written in English and with the following study designs were included; cross-sectional, case control, cohorts or randomized controlled trials.

We excluded postmortem studies and whole-body MRI used for body fat composition, body stature or muscle mass quantification. Clinical studies using whole-body MRI to evaluate fat infiltration of muscle, e.g. in neuromuscular diseases, and studies using whole-body MRI angiography or whole-body integrated MRI/positron emission tomography (PET) were also excluded.

\section{Data and variables}

Information on study and patient characteristics, including methodology and indications for performing whole-body MRI and the imaging protocol with technical parameters, were extracted from the included studies. The following technical parameters were registered: field strength, sequences used, scan plane, slice thickness, repetition time (TR) and echo time (TE) for T1weighted (T1-W), fluid sensitive sequences (T2-weighted/short tau inversion recovery [STIR]) and diffusion-weighted imaging (DWI) and inversion time for STIR sequences and b-values for DWI. If possible, acquired voxel volumes were extracted, either directly or calculated. The voxel size was calculated from the field of view (FOV) and the matrix, using a scan percentage of $100 \%$. We sought to extract the acquired voxel volumes, as a measure for others to repeat the studies. When the FOV was given as two different values, the highest number was used to calculate the voxel size in the frequency direction. When the FOV was given as a range, the mean value was used. We registered the use of contrast, sedation and anatomical coverage. For T2-weighted (T2-W), STIR and DWI sequences, we registered whether motion artefact reduction methods were used. We also assessed the variation in total scan time and the use of audio or video during scanning. All included studies were checked for information about intra- and interobserver agreement and if imaging findings were validated. In addition, we registered papers that mentioned clinically silent lesions (related to expected pathology) and incidental findings (unrelated to known or expected pathology), and whether the possibility of these findings being normal variants was discussed. Also registered was whether the use of whole-body MRI affected mortality and/or morbidity. We also investigated if some studies attempted to establish a standard 
reporting or scoring system for whole-body MRI. Finally, we registered if a radiologist was amongst the authors and in which journals the papers were published.

If some of the information we sought was unclear, we tried to find the missing information from images or figure legends. When available, supplemental material was read, and additional information was extracted. Information about the scan parameters and resolution was checked against the paper text. The values were not registered if the information was contradictory.

\section{Statistics}

Data were exported into SPSS (Statistical Package version 26; IBM Corp., Armonk, NY) and cleaned. Frequencies were given as numbers, with appropriate measures such as percent, proportions or ratios.

\section{Results}

After removing duplicates, 1,357 publications were screened by reading titles and abstracts. The 128 judged possibly eligible were read in full text. Of the 54 papers included $[1-3,5,6$, 27-75] (Fig. 1), 78\% were published during 2010-18 (Fig. 2). The majority of included studies $(98.2 \%)$ focused mainly of children and adolescents (up to 18 years old). Forty papers (74.1\%) were exclusively about children and adolescents, and in 13 papers $(24.1 \%)$ the focus was on both children/ adolescents and adults, but the majority of patients were still younger than 19 years old. The majority of participants were adults in only one of the included papers [42].

\section{Study and patient characteristics}

Thirty out of the 54 studies (55.6\%) had a retrospective design whilst $24(44.4 \%)$ were prospective. None of the included studies was a randomised clinical trial. The number of individuals who underwent one or more whole-body MRI in each included paper varied from 7 to 82 (mean: 26, standard deviation [SD] 17). The mean age was given in 38 papers, and it ranged from 0 to 19 years; in 24 papers $(60.5 \%)$, it ranged from 10 to 15 years. The studies included 1,829 whole-body MRI examinations (mean: 33, SD 22). Fifteen studies (27.8\%) reported on protocols adjusted to child size, indication, vendor or field strength, and these protocols were examined separately.

\section{Indications}

Based on the included studies, we found chronic nonbacterial osteomyelitis (15 papers), lymphoma ( 8 papers) and
Fig. 1 Flow diagram for study exclusion and inclusion

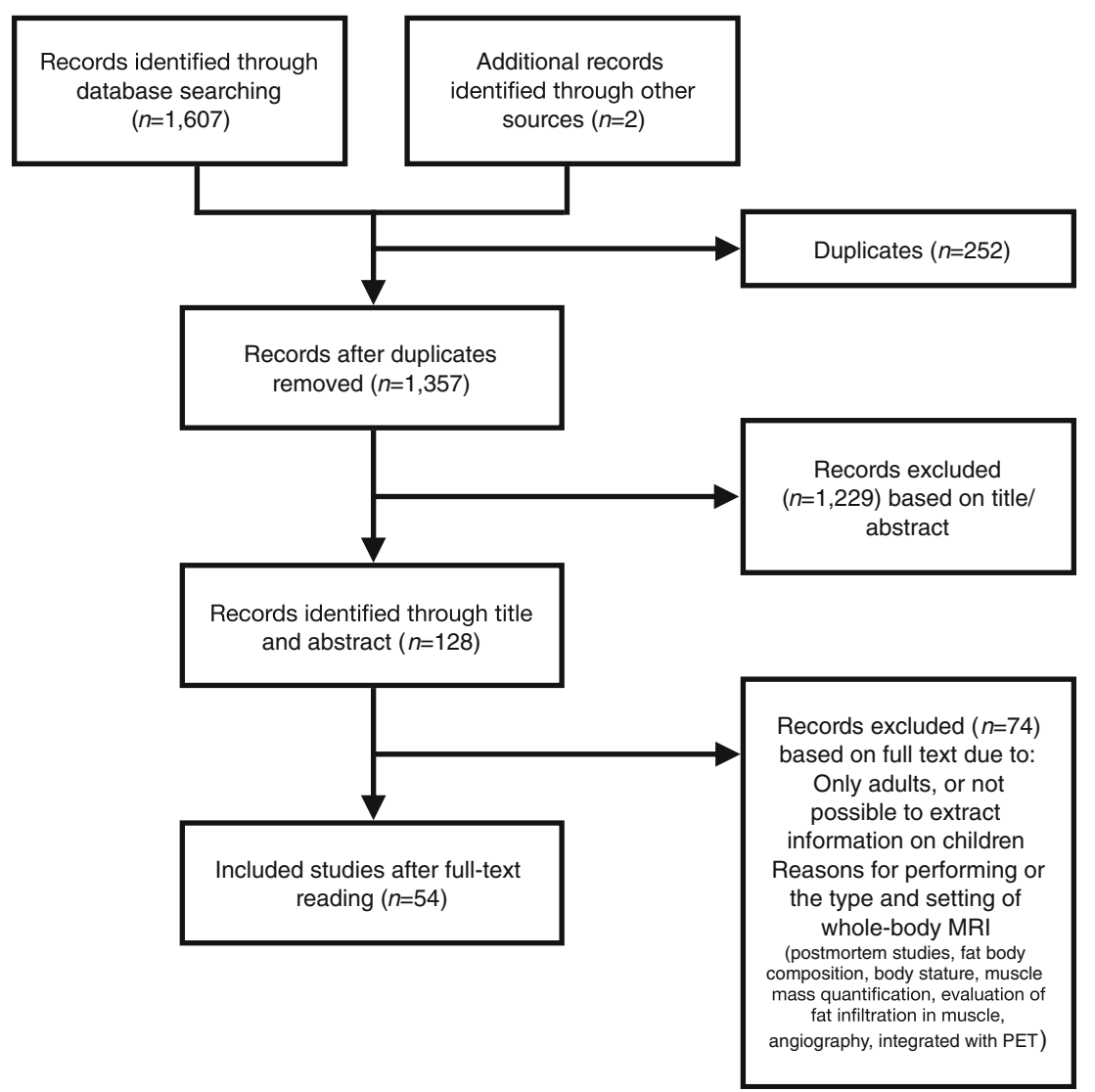


Fig. 2 Included papers by year of publication
9

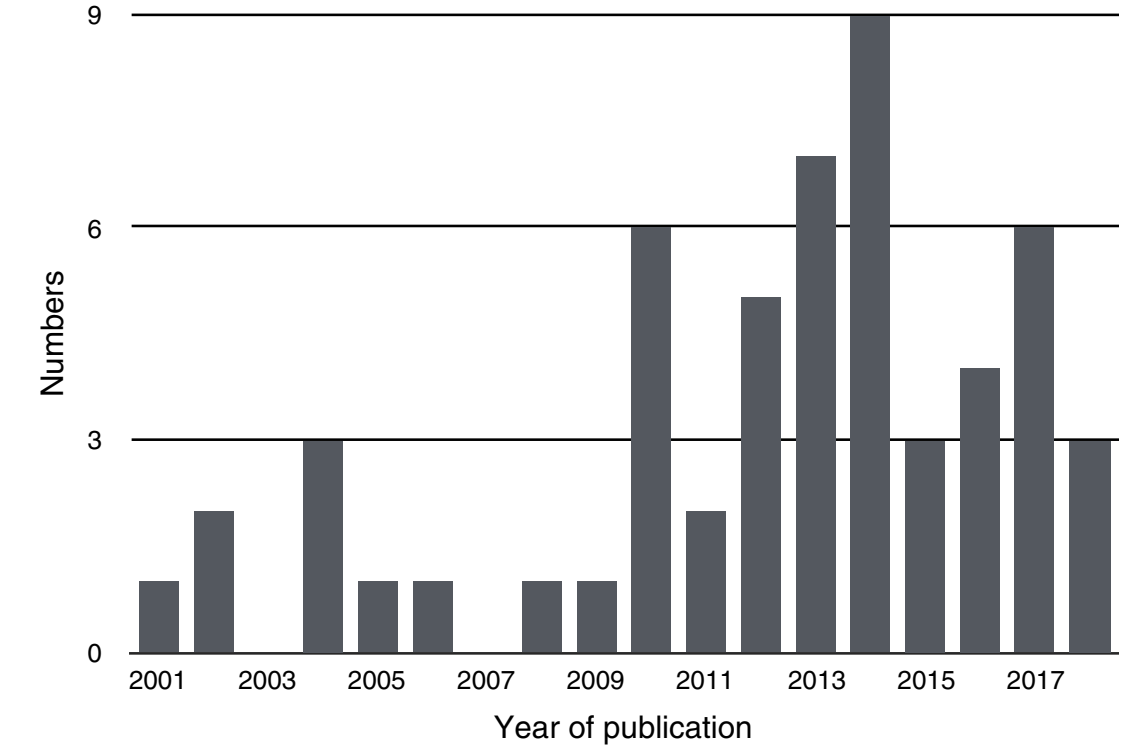

cancer staging ( 8 papers) to be the most frequent indications for performing a whole-body MRI. All examinations had the purpose of diagnosing and/or evaluating the extent of disease. In six papers, the indications for the study participants to undergo whole-body MRI differed, and were consequently registered as multiple indications (Table 1). One paper, for example, included cases of suspected child abuse, chronic nonbacterial osteomyelitis, lymphoma, osteosarcoma, neuroblastoma and Langerhans cell histiocytosis [59].

Table 1 Indications for performing whole-body MRI in studies included in our review

\begin{tabular}{ll}
\hline Indication & $\begin{array}{l}\text { Number of } \\
\text { papers }\end{array}$ \\
\hline Chronic nonbacterial osteomyelitis & 15 \\
Lymphoma & 8 \\
Metastasis & 8 \\
Cancer predisposition syndrome disease & 4 \\
Juvenile dermatomyositis & 2 \\
Osteonecrosis & 3 \\
Neurofibromatosis & 3 \\
Langerhans cell histiocytosis & 1 \\
Nonaccidental injury & 1 \\
Healthy term infants & 1 \\
Sickle cell disease & 1 \\
Acute myeloid leukemia & 1 \\
Multiple indications in the same paper (i.e. cystic & 6 \\
$\quad$ angiomatosis, Langerhans cell histiocytosis, lymphoma, & \\
neuroblastoma, rhabdomyosarcoma, Ewing tumor, & \\
$\quad$ chronic nonbacterial osteomyelitis, suspected child & \\
abuse, etc.) & \\
\hline
\end{tabular}

\section{Field strength}

Of the 54 studies, 34 (63\%) used a 1.5-tesla (T) system only, while 6 (11\%) used $3 \mathrm{~T}$ only. Three of the 54 studies (5.5\%) used both $1.5 \mathrm{~T}$ and $3 \mathrm{~T}$. Eleven studies did not give any information on field strength.

\section{Protocol}

The typical whole-body MRI protocol included STIR sequences only (20 papers) [29-32, 38, 39, 41-44, 51, 56, $58,61,63,66-68,74,75]$ or STIR and T1-W sequences (17 papers) $[2,3,5,27,28,35,37,40,45,46,49,50,55,57,60$, $62,71]$. Five studies used a protocol including STIR, T1-W and DWI [36, 54, 59, 64], and four studies used only STIR and DWI $[52,53,69,70]$. In one study, whole-body MRI was performed on equipment from different vendors with different protocols; the use of STIR was mentioned, but no information about the use of T1-W or DWI was given [73]. In two studies, the term STIR only appeared in a legend, and no information was given on whether the study included T1-W or DWI [1, $72]$. In two of the studies, information on sequences was unavailable $[6,65]$.

\section{Radiofrequency coils}

In 9 studies, the body coil was used as the receiver coil. In 18 studies, dedicated surface coils were used. Twenty-seven papers did not give information on coil type.

\section{Technical settings}

Forty-nine out of 54 studies (90.7\%) used fluid-sensitive sequences, of which 48 used STIR. One of the studies used a 
single-shot T2 sequence and three papers did not report on whether a fluid-sensitive sequence had been used. Thirtynine of the 48 studies using STIR performed the scan in the coronal plane. One study examined protocols from different institutions, and T2 Dixon/T2 fat saturated was mentioned as an alternative to STIR, although this was used in only a few cases [73]. FOV in the feet-head direction varied from 200 to $500 \mathrm{~mm}$ per sequence. For papers providing detailed information on the technical settings, TR, TE, STIR inversion time and slice thickness varied substantially (Table 2). Two of the 48 studies $(4.2 \%)$ using STIR sequences gave the acquired voxel volume, and for 14 studies $(29.2 \%)$, this parameter could be calculated from the FOV and the matrix (as described in the Materials and methods section).

A T1-W sequence was included in 24 studies $(44.4 \%)$, of which $15(62.5 \%)$ were performed in the coronal plane. FOV in the feet-head direction varied from 265 to $500 \mathrm{~mm}$. Fourteen of the 24 studies $(58.3 \%)$ used fast spin-echo sequences and the TR, TE and slice thickness varied greatly amongst these studies (Table 2). In two studies, T1 was performed as a gradient echo sequence. One of the studies (7.1\%) using fast spin-echo sequences reported the acquired voxel volume; for 6 studies (42.9\%), it was calculated.

Ten of the 54 studies (18.5\%), all of which were published after 2012, included a DWI sequence. For 8 of the 10 studies, DWI was performed in the axial plane. We could not assess information about the scan plane in the two remaining papers. In 1 of the 10 papers, the acquired voxel volume was given and in 3 it could be calculated from the parameters (Table 2).
All 10 studies used more than $1 \mathrm{~b}$-value and the highest bvalue was 1,000 .

\section{Contrast enhancement}

Five of the included studies used contrast-enhanced sequences as part of the whole-body MRI protocol [5, 37, 40, 47, 62]. A sixth study used contrast, but it was unclear whether this was part of the whole-body protocol [71]. Five of the six used gadolinium and one study used iron oxide nanoparticles [47].

\section{Sedation and audio/video}

In 16 of the 54 studies (29.6\%), sedation was given. Eleven studies did not use sedation and in 27 papers $(50 \%)$ this information was not provided. None of the included papers reported the use of audio or video during the whole-body MRI examination.

\section{Artefact reduction techniques}

Artefact reduction techniques were used in 1 of the 10 protocols with DWI (10\%) and in 11 protocols with fluid sensitive sequences $(22.4 \%)$. The different artefact reduction techniques were breath-hold and respiratory triggering, electrocardiographic gating and buscopan as an antispasmodic agent.

Table 2 Technical parameters at $1.5 \mathrm{~T}$ and $3 \mathrm{~T}$ used for whole-body MR performed in children and adolescents for various indications, based on a systematic review of the literature up to November 2018

\begin{tabular}{|c|c|c|c|c|c|c|}
\hline \multirow[b]{2}{*}{ Parameters } & \multicolumn{3}{|c|}{$\begin{array}{l}1.5 \mathrm{~T} \\
\text { Range (mean) (SD) (number of papers) }\end{array}$} & \multicolumn{3}{|c|}{$\begin{array}{l}3 \mathrm{~T} \\
\text { Range (mean) (SD) (number of papers) }\end{array}$} \\
\hline & T2-weighted/STIR ${ }^{\mathrm{a}}$ & $\begin{array}{l}\text { T1-weighted } \\
\text { fast spin echo }\end{array}$ & Diffusion-weighted & T2-weighted/STIR ${ }^{\mathrm{a}}$ & $\begin{array}{l}\text { T1-weighted fast } \\
\text { spin echo }\end{array}$ & Diffusion-weighted \\
\hline $\begin{array}{l}\text { Repetition } \\
\text { time (ms) }\end{array}$ & $\begin{array}{c}800-8,500(3,897.3) \\
(2,014.5)(29)\end{array}$ & $\begin{array}{c}85-1,610(608.5) \\
\quad(394.8)(10)\end{array}$ & $\begin{array}{l}4,500-8,612 \\
\quad(6,892.1)(1,602.3) \\
\quad(6)\end{array}$ & $\begin{array}{c}2000-9,126(4664) \\
(2,033.2)(6)\end{array}$ & $\begin{array}{l}480-820(694.3) \\
\quad(186.5)(2)\end{array}$ & $\begin{array}{l}6,100-7,200 \\
\quad(6,566.7)(568.6) \\
\quad(2)\end{array}$ \\
\hline Echo time (ms) & $\begin{array}{l}17-284(65.1)(52.9) \\
\quad(29)\end{array}$ & $\begin{array}{l}7-18(12)(2.9) \\
\quad(10)\end{array}$ & $62-78(72.6)(6.2)(6)$ & $\begin{array}{l}30-326(87.9) \\
(98.2)(6)\end{array}$ & $\begin{array}{l}9-12(10.3)(1.5) \\
\quad(2)\end{array}$ & $55-62(58.5)(5)(2)$ \\
\hline $\begin{array}{l}\text { STIR inversion } \\
\text { time (ms) }\end{array}$ & $\begin{array}{l}64-180(148.4) \\
\quad(21.2)(27)\end{array}$ & - & - & $160-230(205)(5)$ & - & - \\
\hline $\begin{array}{l}\text { Slice thickness } \\
(\mathrm{mm})\end{array}$ & $\begin{array}{l}1.3-10(5.7)(1.8) \\
\quad(29)\end{array}$ & $\begin{array}{l}4-9.5(5.9)(1.8) \\
\quad(10)\end{array}$ & $4-6(4.3)(0.8)(6)$ & $3-8(5.2)(1.6)(6)$ & $\begin{array}{l}4-5.5(4.8)(0.8) \\
\quad(2)\end{array}$ & $4-4.5(4.3)(0.4)(2)$ \\
\hline $\begin{array}{l}\text { Acquired voxel } \\
\text { volume }\left(\mathrm{mm}^{3}\right)\end{array}$ & $\begin{array}{l}2.7-45(18.4)(13.2) \\
\quad(14)\end{array}$ & $\begin{array}{l}7.5-45(21.8) \\
\quad(14.6)(6)\end{array}$ & $\begin{array}{l}19.2-82.8(57.6) \\
\quad(23.3)(3)\end{array}$ & $\begin{array}{l}7.8-11.3(9.1)(1.9) \\
\text { (3) }\end{array}$ & $6.1^{\mathrm{b}}$ & $15^{\mathrm{b}}$ \\
\hline
\end{tabular}




\section{Scan time}

Scan time was defined differently in the studies and varied between sequence time, total MR machine time and time spent in the department. It was not possible to extract sufficient information about the length of the individual sequences used.

\section{Body coverage}

The whole body from head to toe was included in 32 studies $(59.3 \%)$. In nine of the papers, no information on coverage was given. In the remaining studies $(24.1 \%)$, the body coverage varied from head to pelvis or head to thigh.

\section{Reliability and validation of findings}

Ten of the studies $(18.5 \%)$ performed repeatability tests, of which 2 studies assessed inter- and intra-observer agreement $[32,56]$ and 8 examined for interobserver agreement only $[5$, $38,47,52,54,59,61,67]$. Their results varied depending on what was assessed from the whole-body MRI. In the two studies assessing inter- and intra-observer reliability, the intra-observer agreement was higher than the agreement between the readers. Two studies looked at interobserver agreement for both nodal and extranodal sites, and scoring of the nodal sites had a better agreement than the extranodal, including bone marrow lesions [52, 54]. Thirty-nine studies $(72.2 \%)$ did not perform repeatability tests and in 5 of the 54 included studies there was no information on inter- and intra-observer agreement. Thirty-two studies (59.3\%) compared whole-body MRI findings with an established reference standard, i.e. histology/biopsy (reference standard) and/or observation including biopsy and follow-up imaging. Eighteen studies (33.3\%) did not validate their findings. In four studies, no information on validation was given. None of the studies performed biopsy of all lesions that were scored as pathology on whole-body MRI.

\section{Interpretation/reporting system}

Several of the papers included in this review described the imaging criteria they used to define pathology. However only one paper, from 2017 by Arnoldi et al. [28] proposed a standardized reporting system for whole-body MRI for patients with chronic nonbacterial osteomyelitis.

\section{Incidental findings, clinically silent lesions and normal variants}

Six papers reported on incidental MR findings $[5,27,29,33$, 36, 64]. In the study from Anupindi et al. [27], 55 incidental findings were detected in 23 of 24 patients $(96 \%)$. None of the findings was of significant clinical impact requiring imaging follow-up or treatment. The remaining five papers did not provide any details on incidental findings.

In 18 of the papers (33.3\%), the authors concluded that whole-body MRI is a sensitive tool for detecting clinically silent lesions $[1-3,5,6,28,32,35,37,40,43,46,56,60$, $62,72,73,75]$, of which only 3 papers discussed the possibility of these findings being due to normal growth related changes [35, 37, 75]. In total, 12 of 54 papers $(22.2 \%)$ suggested bone bruise and/or highly cellular bone marrow as a cause of high signal on T2-W sequences $[34,35,37,45,50$, $51,53,57-59,66,75]$. One paper mentioned in a figure legend that diffuse high signal of the bone marrow at DWI is a normal finding in the spine and pelvis in children [54].

\section{Effect on long-term disease course}

None of the included studies addressed whether whole-body MRI affected long-term disease course (mortality/morbidity).

\section{Authors and journals}

Fifty of the 54 papers had a radiologist amongst the authors, in 30 of these (55.6\%) as a first author. Sixteen papers (32.7\%) contained sufficient technical parameters for the reader to reproduce the T2-W/STIR resolution, whereof $14(87.5 \%)$ had a radiologist as the first author and were published in radiology journals. In total, 30 of the included papers $(55.6 \%)$ were published in radiology journals.

\section{Discussion}

Our results show that whole-body MRI is commonly used in many institutions, particularly to assess chronic nonbacterial osteomyelitis, lymphoma and malignancy/metastasis. However, the body area covered, the sequences used and the technical details vary significantly. More importantly, only a few studies address the validity of MRI findings.

We found that the term "whole-body MRI" is used interchangeably for examinations covering from two-thirds to the whole body. For instance, whole-body MRI performed for staging of malignant disease sometimes covers skull-base to mid-thigh only [42, 52, 54, 67-69], similar to what is often being used for PET-CT. According to some authors, important additional clinical information, like therapy-related osteonecrosis of the knees/ankles [55] or distant metastases in the extremities [73] could be missed due to this limited FOV, hence a clear definition of what should be covered in the scan must be defined when recommending whole-body MRI for specific diseases. To standardize the nomenclature, we agree with Greer et al. [25] in defining whole-body MRI as a "multiregional contiguous imaging of the entire body" and arguing that the term "whole-body MRI" should be reserved 
for "head-to-feet or vertex-to-heel imaging unless otherwise specified" [23]. Moreover, Greer et al. [23] states that "where smaller FOV is sufficient, whole-body MRI can be truncated and should be annotated accordingly."

The current literature does not allow us to give any preferences on field strength for whole-body MR imaging in children, i.e. whether a $3-\mathrm{T}$ system performs better than a $1.5-\mathrm{T}$ system. This issue was addressed in one study only including 21 children with different tumors, examined on both 1.5-T and 3-T systems [61]. They found substantial agreement between two observers, but a better image quality on the $1.5 \mathrm{~T}$, and also fewer artefacts. They concluded that this difference was unlikely to be significant as the image quality of the 3-T protocol was scored high enough. It is, however, difficult to compare field strength as such because the resultant image also depends on the machine hardware, software, coil and the protocol used.

Most protocols included coronal STIR and T1-W sequences, but the technical settings varied considerably across institutions. The current use of STIR sequences was not unexpected, as this is a particularly robust, widely used imaging method that may improve lesion detection. However, recent technical improvements have resulted in methods with better signal to noise ratios, such as $\mathrm{T} 2-\mathrm{W}$ with spectral fat saturation and T2-W Dixon, allowing for higher image resolution. In the present review, only one study mentioned T2-W Dixon, however, with no elaboration on a potentially added value [73]. The Dixon technique provides images with and without fat suppression and fat only images, which may replace the T1W fast spin echo (FSE) images for bone marrow pathology [76]. This may reduce examination time, which is particularly beneficial in children.

One might argue that varying technical settings for the different pulse sequences, such as TR, TE and image resolution, is inevitable due to different scanners, indications, coils, body sizes, etc., and that especially the TR of T2-W/STIR sequences often is irrelevant. However, we found that TR and TE times of T2-W/STIR sequences varied significantly (Table 2), and to such an extent that it might influence image quality and, more importantly, image analysis. A short TR of $800 \mathrm{~ms}$ in a T2-W sequence can result in low signal-to-noise ratio and additional $\mathrm{T} 1$ weighting, while a short TE of $17 \mathrm{~ms}$ in a $\mathrm{T} 2-\mathrm{W}$ sequence gives less $\mathrm{T} 2$ and more proton weighting. The upper range of TE for the T2-W/STIR sequences was higher than expected and could result in low signal-to-noise ratio. This would reduce image resolution and, consequently, decrease lesion detection. Further, variation in STIR inversion time influences the degree of fat suppression, whilst high TR times in T1 sequences minimize the T1 weighting. We believe that defining a size- or weightbased standard protocol for whole-body MRI within institutions works for most practical purposes and indications. We also believe that defining standard and/or minimum requirements for technical settings, e.g., maximum slice thickness
(3-4 mm depending on patient size or weight) across institutions to ensure sufficient diagnostic quality and to perform comparative studies, is warranted.

The use of DWI as part of the whole-body MRI has been addressed in several studies on adults, however, only 10 of the current studies included a DWI sequence. Five of the studies assessed a potential added value of DWI in addition to anatomical sequences. Three of these studies concluded that, in contrast to what has been shown for adults, there was no significant benefit in diagnostic accuracy from adding DWI sequences $[52,54,59]$. According to the authors, this could be related to the small number of patients in the current studies or to child-specific issues such as smaller body-size or challenges in interpretation of bone marrow signal in children due to inhomogeneous DWI signal. Two papers concluded that DWI could add useful information [53,69]. The study by Leclair et al. [53] showed a substantial elevation of the apparent diffusion coefficient (ADC) values in inflammatory bone lesions due to chronic recurrent osteomyelitis (chronic nonbacterial osteomyelitis). Hence, the authors highlighted DWI as a promising technique that may help to distinguish benign inflammatory processes from malignant lesions. However, they only assessed chronic nonbacterial osteomyelitis lesions. Furthermore, they also mentioned the limitations of DWI in children due to high bone marrow signals. Punwani et al. [69] investigated the added value of DWI to PET to predict local treatment response in children and adolescents with Hodgkin lymphoma. Pretreatment ADC values in nodal masses with adequate response were significantly lower than in sites with inadequate response. Based on their results, the authors suggested that DWI should be incorporated in integrated PET/MRI protocols [69].

This review revealed inconsistent, and often suboptimal, reporting of MR technical settings. Most often, a combination of FOV, imaging matrix and slice thickness was provided. For some protocols, ranges of FOV and slice thickness were given, as these parameters were adjusted, according to the region scanned. Numerous studies lacked sufficient data for calculating image resolution, making it impossible for the reader to reproduce the protocol/images. In our opinion, details to assess the acquired voxel size should be provided routinely when reporting on whole-body MRI. The acquired voxel size was reported in only a few papers, while from some papers the reader could deduce the acquired voxel size based on given parameters, and by an assumption of the scan percentage. We used a scan percentage of $100 \%$ to calculate the in-plane resolution, although it is more common to use a scan percentage of around $80 \%$ since this reduces scan time. However, this means that our calculated voxel sizes can be slightly smaller than what was used in the protocol, and underestimation of the resolution was thus avoided.

The voxel size used for the T2-W/STIR sequences varied by a factor of 20 . None of the studies, however, compared the 
effect of different voxel sizes on image quality, or, more importantly, on the imaging findings. One study, however, proposed a high-resolution 3-D STIR scan as the sole sequence, and compared this to a protocol with both 3-D STIR, T1 and DWI [59] concluding that the protocols were equally good at detecting pathology. The technical improvements of MR equipment, software and coils make it increasingly feasible to image with higher resolution, although we found no significant trend when comparing voxel size and year of publication. It is possible that some sites would rather perform more sequences, acquisitions in more imaging planes, or shorten total scan time, rather than choosing a higher resolution scan. We were not able to extract enough information on actual scan times.

Detailed information on the coils used was sparse. Some authors used a body coil. Although comfortable for the child, this coil gives a low signal-to-noise ratio, thus hindering highresolution imaging. Dedicated coils, such as phased array coils allowing for high resolution imaging, were used in several of the studies. The variety of coils used may, in part, explain the wide range of image resolution found for all types of sequences.

Only a few studies reported on child-specific issues such as the use of sedation and/or motion artefact reducing methods, be it technical or practical such as watching films or listening to music. For papers by clinicians in non-radiologic journals this is understandable, however, many of the radiology-driven studies also lacked this important information enabling others to reproduce the examination.

In the adult literature, several studies report on the frequency of incidental whole-body MRI findings [10, 77, 78], but in children this information is sparse. In the current review, only one study addressed this issue, reporting a prevalence of $96 \%$ [27]. In general, there is no published consensus on how to manage incidental findings on wholebody MRI [10]. Moreover, due to the lack of normal reference standards for whole-body MRI in children, it may be difficult to define the findings that have "potential health or reproductive importance" [79].

Amongst the papers included in the current review, several concluded that the sensitivity of whole-body MRI for detecting bone-marrow lesions is superior to that of conventional imaging methods $[2,34,38,62,70]$. Further, several authors emphasized the method's ability to detect clinically silent lesions, thus without discussing the risk of false-positive findings due to growth-related signal variation. Amongst these were two papers describing any bone marrow hyperintensities as either related to disease [41] or therapy [43] without giving their definition of pathology.

The lack of standardized interpretation of whole-body MRI findings may lead to misinterpretation and subsequent consequences for diagnosis and treatment. This is illustrated by the study of Zibrowska-Bech et al. [3], who retrospectively evaluated 31 children diagnosed with chronic nonbacterial osteomyelitis over 10 years [3]. One of the children in the study was erroneously treated with chemotherapy for 2 weeks under the suspicion of having osteosarcoma, before the diagnosis was changed to chronic nonbacterial osteomyelitis. Another child had initially been misdiagnosed with Langerhans cell histiocytosis and was treated with vinblastine and prednisolone for 6 months before a chronic nonbacterial osteomyelitis diagnosis. In both cases, there was a substantial diagnostic delay, ranging from 8.4 months in the first case to 37 months in the second case.

Only a few studies performed reliability tests, but both papers reporting on inter- and intra-observer agreement showed a substantially higher score for intra-observer agreement, in part reflecting the lack of definitions for MRI pathology. Opposite, the interobserver agreement for evaluating nodal versus extranodal pathology, clearly defined by international standards, was good $[52,54]$.

Histology was used in 17 studies to validate the accuracy of the whole-body MRI findings. For obvious reasons, not all findings on MRI could be biopsied, hence some of the lesions detected on whole-body MRI could still be normal findings or unrelated to the underlying disease.

There are several limitations to this review. Important information from non-English publications or from studies by the clinical site without indexing "whole-body MRI" may have been missed. Another potential bias is the exclusion of combined adult-paediatric studies with insufficient information on the included children/adolescents. It was not possible to extract information about average scan time due to a lack in data. Lastly, studies listing multiple indications were registered as "multiple," thus decreasing specificity.

\section{Conclusion}

Whole-body MRI is being used in the pediatric population for a wide variety of reasons. Chronic nonbacterial osteomyelitis, lymphoma and metastasis are amongst the most common indications. There is no consensus on a standardized imaging protocol, and due to insufficient reporting on technical data, a reproduction of the protocol or images from the published literature is hardly possible. Furthermore, studies addressing the precision, accuracy and clinical validity of whole-body MRI in children are lacking and, to date, there is no documented effect on morbidity and/or mortality. The results from our study could potentially be used to boost attempts toward standardization of technique, reporting and guidelines development.

Acknowledgements Open Access funding provided by UiT The Arctic University of Norway. 


\section{Compliance with ethical standards}

\section{Conflicts of interest None}

Open Access This article is licensed under a Creative Commons Attribution 4.0 International License, which permits use, sharing, adaptation, distribution and reproduction in any medium or format, as long as you give appropriate credit to the original author(s) and the source, provide a link to the Creative Commons license, and indicate if changes were made. The images or other third party material in this article are included in the article's Creative Commons license, unless indicated otherwise in a credit line to the material. If material is not included in the article's Creative Commons license and your intended use is not permitted by statutory regulation or exceeds the permitted use, you will need to obtain permission directly from the copyright holder. To view a copy of this license, visit http://creativecommons.org/licenses/by/4.0/.

\section{References}

1. Beck C, Morbach H, Beer M et al (2010) Chronic nonbacterial osteomyelitis in childhood: prospective follow-up during the first year of anti-inflammatory treatment. Arthritis Res Ther 12:R74

2. Guerin-Pfyffer S, Guillaume-Czitrom S, Tammam S, Kone-Paut I (2012) Evaluation of chronic recurrent multifocal osteitis in children by whole-body magnetic resonance imaging. Joint Bone Spine 79:616-620

3. Ziobrowska-Bech A, Fiirgaard B, Heuck C et al (2013) Ten-year review of Danish children with chronic non-bacterial osteitis. Clin Exp Rheumatol 31:974-979

4. Hofmann C, Wurm M, Schwarz T et al (2014) A standardized clinical and radiological follow-up of patients with chronic nonbacterial osteomyelitis treated with pamidronate. Clin Exp Rheumatol 32:604-609

5. Yoon HM, Kim JR, Jung AY et al (2017) Whole body MR imaging: a useful imaging modality in the management of children with acute myeloid leukemia. Clin Lymphoma Myeloma Leuk 17:231237

6. Schnabel A, Range U, Hahn G et al (2016) Unexpectedly high incidences of chronic non-bacterial as compared to bacterial osteomyelitis in children. Rheumatol Int 36:1737-1745

7. Albano D, Agnello F, Patti C et al (2017) Whole-body magnetic resonance imaging and FDG-PET/CT for lymphoma staging: assessment of patient experience. Egypt J Radiol Nucl Med 48:10431047

8. Weber U, Hodler J, Kubik RA et al (2009) Sensitivity and specificity of spinal inflammatory lesions assessed by whole-body magnetic resonance imaging in patients with ankylosing spondylitis or recent-onset inflammatory back pain. Arthritis Rheum 61:900-908

9. Michielsen K, Vergote I, Op de Beeck K et al (2014) Whole-body MRI with diffusion-weighted sequence for staging of patients with suspected ovarian cancer: a clinical feasibility study in comparison to CT and FDG-PET/CT. Eur Radiol 24:889-901

10. Hegenscheid K, Seipel R, Schmidt CO et al (2013) Potentially relevant incidental findings on research whole-body MRI in the general adult population: frequencies and management. Eur Radiol 23:816-826

11. Avenarius DFM, Ording Müller LS, Rosendahl K (2017) Joint fluid, bone marrow edemalike changes, and ganglion cysts in the pediatric wrist: features that may mimic pathologic abnormalitiesfollow-up of a healthy cohort. AJR Am J Roentgenol 208:13521357
12. Avenarius DF, Ording Müller LS, Rosendahl K (2016) Erosion or normal variant? 4-year MRI follow-up of the wrists in healthy children. Pediatr Radiol 46:322-330

13. Avenarius DM, Ording Müller LS, Eldevik P et al (2012) The paediatric wrist revisited-findings of bony depressions in healthy children on radiographs compared to MRI. Pediatr Radiol 42:791798

14. Ording Müller LS, Avenarius D, Damasio B et al (2011) The paediatric wrist revisited: redefining MR findings in healthy children. Ann Rheum Dis 70:605-610

15. Ording Müller LS, Avenarius D, Olsen OE (2011) High signal in bone marrow at diffusion-weighted imaging with body background suppression (DWIBS) in healthy children. Pediatr Radiol 41:221226

16. Ording Müller LS, Boavida P, Avenarius D et al (2013) MRI of the wrist in juvenile idiopathic arthritis: erosions or normal variants? A prospective case-control study. Pediatr Radiol 43:785-795

17. Shabshin N, Schweitzer ME, Morrison WB et al (2006) High-signal T2 changes of the bone marrow of the foot and ankle in children: red marrow or traumatic changes? Pediatr Radiol 36:670-676

18. Smets AM, Deurloo EE, Slager TJE et al (2018) Whole-body magnetic resonance imaging for detection of skeletal metastases in children and young people with primary solid tumors - systematic review. Pediatr Radiol 48:241-252

19. Eutsler EP, Khanna G (2016) Whole-body magnetic resonance imaging in children: technique and clinical applications. Pediatr Radiol 46:858-872

20. Schooler GR, Davis JT, Daldrup-Link HE, Frush DP (2018) Current utilization and procedural practices in pediatric wholebody MRI. Pediatr Radiol 48:1101-1107

21. Chavhan GB, Babyn PS (2011) Whole-body MR imaging in children: principles, technique, current applications, and future directions. Radiographics 31:1757-1772

22. Gottumukkala RV, Gee MS, Hampilos PJ, Greer MC (2019) Current and emerging roles of whole-body MRI in evaluation of pediatric cancer patients. Radiographics 39:516-534

23. Greer MLC, Voss SD, States LJ (2017) Pediatric Cancer predisposition imaging: focus on whole-body MRI. Clin Cancer Res 23:e6e13

24. Goo HW (2015) Whole-body MRI in children: current imaging techniques and clinical applications. Korean J Radiol 16:973-985

25. Greer MC (2018) Whole-body magnetic resonance imaging: techniques and non-oncologic indications. Pediatr Radiol 48:13481363

26. Damasio MB, Magnaguagno F, Stagnaro G (2016) Whole-body MRI: non-oncological applications in paediatrics. Radiol Med 121:454-461

27. Anupindi SA, Bedoya MA, Lindell RB et al (2015) Diagnostic performance of whole-body MRI as a tool for cancer screening in children with genetic cancer-predisposing conditions. AJR Am J Roentgenol 205:400-408

28. Arnoldi AP, Schlett CL, Douis H et al (2017) Whole-body MRI in patients with non-bacterial osteitis: radiological findings and correlation with clinical data. Eur Radiol 27:2391-2399

29. Bueno MT, Martinez-Rios C, la Puente GA et al (2017) Pediatric imaging in DICER1 syndrome. Pediatr Radiol 47:1292-1301

30. Burdach S, Thiel U, Schoniger M et al (2010) Total body MRIgoverned involved compartment irradiation combined with highdose chemotherapy and stem cell rescue improves long-term survival in Ewing tumor patients with multiple primary bone metastases. Bone Marrow Transplant 45:483-489

31. Castro TC, Lederman H, Terreri MT et al (2014) Whole-body magnetic resonance imaging in the assessment of muscular involvement in juvenile dermatomyositis/polymyositis patients. Scand J Rheumatol 4:329-333 
32. Castro TC, Lederman H, Terreri MT et al (2011) The use of jointspecific and whole-body MRI in osteonecrosis: a study in patients with juvenile systemic lupus erythematosus. Br J Radiol 84:621628

33. Clark P, Rooks VJ, Lustik M (2013) Fast whole-body magnetic resonance imaging of healthy, term neonates: spectrum of incidental and birth-related findings. J Comput Assist Tomogr 37:371-376

34. Daldrup-Link HE, Franzius C, Link TM et al (2001) Whole-body MR imaging for detection of bone metastases in children and young adults: comparison with skeletal scintigraphy and FDG PET. Am J Roentgenol 177:229-236

35. Falip C, Alison M, Boutry N et al (2013) Chronic recurrent multifocal osteomyelitis (CRMO): a longitudinal case series review. Pediatr Radiol 43:355-375

36. Friedman DN, Lis E, Sklar CA et al (2014) Whole-body magnetic resonance imaging (WB-MRI) as surveillance for subsequent malignancies in survivors of hereditary retinoblastoma: a pilot study. Pediatr Blood Cancer 61:1440-1444

37. Fritz J, Tzaribatchev N, Claussen CD et al (2009) Chronic recurrent multifocal osteomyelitis: comparison of whole-body MR imaging with radiography and correlation with clinical and laboratory data. Radiology 252:842-851

38. Goo HW, Choi SH, Ghim T et al (2005) Whole-body MRI of paediatric malignant tumours: comparison with conventional oncological imaging methods. Pediatr Radiol 35:766-773

39. Goo HW, Yang DH, Ra YS et al (2006) Whole-body MRI of Langerhans cell histiocytosis: comparison with radiography and bone scintigraphy. Pediatr Radiol 36:1019-1031

40. Hofmann C, Wurm M, Schwarz T et al (2014) A standardised clinical and radiological follow-up of patients with chronic nonbacterial osteomyelitis treated with pamidronate. Clin Exp Rheumatol 32:604-609

41. Hospach T, Langendoerfer M, von Kalle T et al (2010) Spinal involvement in chronic recurrent multifocal osteomyelitis (CRMO) in childhood and effect of pamidronate. Eur J Pediatrics 169:1105-1111

42. Jett K, Nguyen R, Arman D et al (2015) Quantitative associations of scalp and body subcutaneous neurofibromas with internal plexiform tumors in neurofibromatosis 1 . Am J Med Genet A 167: $1518-1524$

43. Karmazyn B, Cohen MD, Jennings SG, Robertson KA (2012) Marrow signal changes observed in follow-up whole-body MRI studies in children and young adults with neurofibromatosis type 1 treated with imatinib mesylate (Gleevec) for plexiform neurofibromas. Pediatr Radiol 42:1218-1222

44. Kellenberger CJ, Miller SF, Khan M et al (2004) Initial experience with FSE STIR whole-body MR imaging for staging lymphoma in children. Eur Radiol 14:1829-1841

45. Kembhavi SA, Rangarajan V, Shah S et al (2014) Prospective observational study on diagnostic accuracy of whole-body MRI in solid small round cell tumours. Clin Radiol 69:900-908

46. Khedr SA, Hassaan MA, Shabana AA et al (2012) Musculoskeletal manifestations of sickle cell disease, diagnosis with whole body MRI. Egypt J Radiol Nucl Med 43:77-84

47. Klenk C, Gawande R, Uslu L et al (2014) Ionising radiation-free whole-body MRI versus (18)F-fluorodeoxyglucose PET/CT scans for children and young adults with cancer: a prospective, nonrandomised, single-Centre study. Lancet Oncol 15:275-285

48. Korchi AM, Hanquinet S, Anooshiravani M, Merlini L (2014) Whole-body magnetic resonance imaging: an essential tool for diagnosis and work up of non-oncological systemic diseases in children. Minerva Pediatr 66:169-176

49. Krohmer S, Sorge I, Krausse A et al (2010) Whole-body MRI for primary evaluation of malignant disease in children. Eur J Radiol $74: 256-261$
50. Kumar J, Seith A, Kumar A et al (2008) Whole-body MR imaging with the use of parallel imaging for detection of skeletal metastases in pediatric patients with small-cell neoplasms: comparison with skeletal scintigraphy and FDG PET/CT. Pediatr Radiol 38:953-962

51. Laffan EE, O'Connor R, Ryan SP, Donoghue VB (2004) Wholebody magnetic resonance imaging: a useful additional sequence in paediatric imaging. Pediatr Radiol 34:472-480

52. Latifoltojar A, Punwani S, Lopes A et al (2018) Whole-body MRI for staging and interim response monitoring in paediatric and adolescent Hodgkin's lymphoma: a comparison with multi-modality reference standard including ${ }^{18}$ F-FDG-PET-CT. Eur Radiol 29: 202-212

53. Leclair N, Thormer G, Sorge I et al (2016) Whole-body diffusionweighted imaging in chronic recurrent multifocal osteomyelitis in children. PLoS One 11:e0147523

54. Littooij AS, Kwee TC, Barber I et al (2014) Whole-body MRI for initial staging of paediatric lymphoma: prospective comparison to an FDG-PET/CT-based reference standard. Eur Radiol 24:11531165

55. Littooij AS, Kwee TC, Enriquez G et al (2017) Whole-body MRI reveals high incidence of osteonecrosis in children treated for Hodgkin lymphoma. Br J Haematol 176:637-642

56. Malattia C, Damasio MB, Madeo A et al (2014) Whole-body MRI in the assessment of disease activity in juvenile dermatomyositis. Ann Rheum Dis 73:1083-1090

57. Mazumdar A, Siegel MJ, Narra V, Luchtman-Jones L (2002) Whole-body fast inversion recovery MR imaging of small cell neoplasms in pediatric patients: a pilot study. AJR Am J Roentgenol 179:1261-1266

58. Mentzel HJ, Kentouche K, Sauner D et al (2004) Comparison of whole-body STIR-MRI and $99 \mathrm{mTc}$-methylene-diphosphonate scintigraphy in children with suspected multifocal bone lesions. Eur Radiol 14:2297-2302

59. Merlini L, Carpentier M, Ferrey S et al (2017) Whole-body MRI in children: would a 3D STIR sequence alone be sufficient for investigating common paediatric conditions? A comparative study. Eur J Radiol 88:155-162

60. Miettunen PM, Lafay-Cousin L, Guilcher GM et al (2012) Widespread osteonecrosis in children with leukemia revealed by whole-body MRI. Clin Orthop Relat Res 470:3587-3595

61. Mohan S, Moineddin R, Chavhan GB (2015) Pediatric whole-body magnetic resonance imaging: intra-individual comparison of technical quality, artifacts, and fixed structure visibility at 1.5 and $3 \mathrm{~T}$. Indian J Radiol Imaging 25:353-358

62. Morbach H, Schneider P, Schwarz T et al (2012) Comparison of magnetic resonance imaging and $99 \mathrm{mTechnetium-labelled} \mathrm{methy-}$ lene diphosphonate bone scintigraphy in the initial assessment of chronic non-bacterial osteomyelitis of childhood and adolescents. Clin Exp Rheumatol 30:578-582

63. Nguyen R, Kluwe L, Fuensterer C et al (2011) Plexiform neurofibromas in children with neurofibromatosis type 1: frequency and associated clinical deficits. J Pediatr 159:652-655.e652

64. O'Neill AF, Voss SD, Jagannathan JP et al (2018) Screening with whole-body magnetic resonance imaging in pediatric subjects with Li-Fraumeni syndrome: a single institution pilot study. Pediatr Blood Cancer 65

65. Pastore S, Ferrara G, Monasta L et al (2016) Chronic nonbacterial osteomyelitis may be associated with renal disease and bisphosphonates are a good option for the majority of patients. Acta Paediatr 105:e328-e333

66. Perez-Rossello JM, Connolly SA, Newton AW et al (2010) Wholebody MRI in suspected infant abuse. AJR Am J Roentgenol 195: 744-750

67. Punwani S, Cheung KK, Skipper N et al (2013) Dynamic contrastenhanced MRI improves accuracy for detecting focal splenic 
involvement in children and adolescents with Hodgkin disease. Pediatr Radiol 43:941-949

68. Punwani S, Taylor SA, Bainbridge A et al (2010) Pediatric and adolescent lymphoma: comparison of whole-body STIR halfFourier RARE MR imaging with an enhanced PET/CT reference for initial staging. Radiology 255:182-190

69. Punwani S, Taylor SA, Saad ZZ et al (2013) Diffusion-weighted MRI of lymphoma: prognostic utility and implications for PET/ MRI? Eur J Nucl Med Mol Imaging 40:373-385

70. Regacini R, Puchnick A, Luisi FAV, Lederman HM (2018) Can diffusion-weighted whole-body MRI replace contrast-enhanced CT for initial staging of Hodgkin lymphoma in children and adolescents? Pediatr Radiol 48:638-647

71. Roderick M, Shah R, Finn A, Ramanan AV (2014) Efficacy of pamidronate therapy in children with chronic non-bacterial osteitis: disease activity assessment by whole body magnetic resonance imaging. Rheumatology 53:1973-1976

72. Roderick MR, Shah R, Rogers V et al (2016) Chronic recurrent multifocal osteomyelitis (CRMO) - advancing the diagnosis. Pediatr Rheumatol Online J 14:47

73. Scheer M, Dantonello T, Brossart P et al (2018) Importance of whole-body imaging with complete coverage of hands and feet in alveolar rhabdomyosarcoma staging. Pediatr Radiol 48:648-657
74. Siegel MJ, Acharyya S, Hoffer FA et al (2013) Whole-body MR imaging for staging of malignant tumors in pediatric patients: results of the American College of Radiology Imaging Network 6660 trial. Radiology 266:599-609

75. von Kalle T, Heim N, Hospach T et al (2013) Typical patterns of bone involvement in whole-body MRI of patients with chronic recurrent multifocal osteomyelitis (CRMO). Rofo 185:655-661

76. Maeder Y, Dunet V, Richard R et al (2018) Bone marrow metastases: T2-weighted Dixon spin-echo fat images can replace T1weighted spin-echo images. Radiology 286:948-959

77. Morin SH, Cobbold JF, Lim AK et al (2009) Incidental findings in healthy control research subjects using whole-body MRI. Eur J Radiol 72:529-533

78. Ulus S, Suleyman E, Ozcan UA, Karaarslan E (2016) Whole-body MRI screening in asymptomatic subjects; preliminary experience and long-term follow-up findings. Pol J Radiol 81:407-414

79. Wolf SM, Lawrenz FP, Nelson CA et al (2008) Managing incidental findings in human subjects research: analysis and recommendations. J Law Med Ethics 36(219-248):211

Publisher's note Springer Nature remains neutral with regard to jurisdictional claims in published maps and institutional affiliations. 